\title{
JNNP
}

\section{Atypical and anaplastic meningiomas: prognostic implications of clinicopathological features}

S-Y Yang, C-K Park, S-H Park, et al.

J Neurol Neurosurg Psychiatry 2008 79: 574-580 originally published online August 31, 2007

doi: 10.1136/jnnp.2007.121582

Updated information and services can be found at:

http://jnnp.bmj.com/content/79/5/574.full.html

Topic collections Articles on similar topics can be found in the following collections

\author{
Immunology (including allergy) (43508 articles) \\ Neurooncology (1036 articles) \\ CNS cancer (654 articles) \\ Radiology (14701 articles) \\ Surgical oncology (6064 articles)
}

Notes

To order reprints of this article go to:

http://jnnp.bmj.com/cgi/reprintform

To subscribe to Journal of Neurology, Neurosurgery \& Psychiatry go to:

http://jnnp.bmj.com/subscriptions 


\title{
Atypical and anaplastic meningiomas: prognostic implications of clinicopathological features
}

\author{
S-Y Yang, ${ }^{1,2}$ C-K Park, ${ }^{2,3}$ S-H Park, ${ }^{4}$ D G Kim, ${ }^{2,3}$ Y S Chung, ${ }^{2,3}$ H-W Jung ${ }^{2,3}$
}

${ }^{1}$ Department of Neurosurgery, DongGuk University International Hospital, Goyang, Korea;

${ }^{2}$ Clinical Research Institute, Seoul National University Hospital, Seoul, Korea:

${ }^{3}$ Department of Neurosurgery, Seoul National University College of Medicine, Seoul, Korea; ${ }^{4}$ Department of Pathology, Seoul National University College of Medicine, Seoul, Korea

Correspondence to: Young Seob Chung, MD, PhD, Department of Neurosurgery, Seoul National University College of Medicine, 28 Yeongeon-dong, Jongno-gu, Seoul 110-744, Republic of Korea; yschung@snu.ac.kr

Received 27 March 2007 Revised 16 August 2007 Accepted 18 August 2007

Published Online First

31 August 2007

\section{ABSTRACT}

Objectives: To evaluate patient outcome and investigate the prognostic factors of high-grade meningiomas by adopting the 2000 World Health Organization (WHO) classification system.

Methods: Between 1986 and 2004, 74 patients were diagnosed with high-grade meningioma: 33 with atypical and 41 with anaplastic meningioma. The mean follow-up was 58.5 months. We reclassified all surgical specimens, according to the 2000 WHO classification system, using two expert neuropathologists.

Results: Forty of 74 meningiomas were reclassified as atypical meningioma and 24 as anaplastic meningioma. Overall and recurrence-free survivals were significantly longer in patients with atypical than in those with anaplastic meningioma: 142.5 versus 39.8 months and 138.5 versus 32.2 months, respectively $(p<0.001)$. In patients with atypical meningiomas, brain invasion and adjuvant radiotherapy were not associated with survival; however, in the brain invasion subgroup, adjuvant radiotherapy improved patients' survival. In patients with anaplastic meningioma, the prognostic factors were brain invasion, adjuvant radiotherapy, malignant progression, p53 overexpression and extent of resection. The p53 overexpression was the only factor associated with malignant progression $(p=0.009)$.

Conclusions: The 2000 WHO classification has identified the truly aggressive meningiomas better than did the previous criteria. A precise meningioma grading system may help to avoid over-treatment of patients with an atypical meningioma as, once the tumour has "declared itself" by recurrence and histological features, it becomes a tumour that is poorly amenable to current therapies.

Since malignant meningioma was first recognised by Cushing and Eisenhardt in 1938, ${ }^{1}$ there have been diverse criteria for histopathologically grading atypical and anaplastic meningiomas. To improve this situation, the 2000 WHO classification recommends much more stringent and objective criteria. ${ }^{2}$ In the 2000 WHO classification, some important diagnostic variables were amended, particularly proliferation index, brain invasion and mitotic activity.

Most tumours tend to become more aggressive in clinical behaviour and more "malignant" in their characteristics over time, although this time course may be variable. Recent studies into the cytogenetic alteration of meningioma have provided tools for understanding the mechanisms underlying malignant progression. ${ }^{3}{ }^{4}$ These advances may be useful in improving our ability to predict clinical outcome and develop therapeutic strategies to improve outcomes in patients with high-grade meningiomas.
This study was primarily motivated by growing concerns about the validity of treatment based on both histological grading and cytogenetic alterations of meningiomas. To address this issue, by adopting the 2000 WHO criteria, we reclassified previous atypical and anaplastic meningiomas, reanalysed their treatment outcomes and re-evaluated prognostic factors by clinicopathological aspects.

\section{MATERIALS AND METHODS \\ Patients}

Between 1986 and 2004, 1098 patients with an intracranial meningioma were treated surgically at Seoul National University Hospital (SNUH). Of these 1098 patients, 79 were previously diagnosed as having atypical or anaplastic meningiomas. The rhabdoid or papillary variants were initially excluded. Five of these 79 patients were also excluded from our study: 1 patient had neurofibromatosis type 2; 1 was younger than 15 years; and biopsy tissue from 3 patients was not available for review. Of the 74 included patients, 33 were originally diagnosed with atypical meningioma and 41 with anaplastic meningioma. Using the 2000 WHO classification system, we reviewed biopsy tissues of these 74 patients.

The clinical records of all patients were reviewed to obtain data on overall survival (time from diagnosis of atypical or anaplastic meningioma to death), recurrence-free survival (time from diagnosis of atypical or anaplastic meningioma to first evidence of recurrence or disease progression either clinically or radiologically), and time for malignant progression (time interval from low-grade meningioma to high-grade meningioma). We also collected the information on both cause of death and date of death through the database of the Korea National Statistical Office and the Korean National Heath Insurance Corporation. The extent of resection was deduced from the operation records or postoperative scans when available, and assessed according to the Simpson grading system. ${ }^{5}$ The institutional review board of SNUH approved this study. However, it did not require informed consent from these patients for their inclusion in this study because the study depended only on information obtained as a part of routine clinical care and patient medical records.

\section{Pathological review}

We reviewed all surgical specimens of the 74 patients, including their previous samples that had been diagnosed as benign meningioma either from SNUH or other hospitals. Two neuropathologists reviewed all available slides according to the 
2000 WHO classification ${ }^{2}$ without prior knowledge of the original grading or patients' outcome. Where differences between the neuropathologists' opinions were encountered, the sections were re-examined by both neuropathologists and results were recorded after an agreement was reached. Brain invasion was recorded as present, absent or unassessable (no adjacent brain parenchyma).

For immunohistochemical studies, the avidin-biotin peroxidase indirect technique with diaminobenzadine as the chromagen was applied using antibody-directed p53 protein (DakoCytomation, Carpinteria, CA, USA; monoclonal, dilution 1:1200) and the proliferation marker MIB-1 (DakoCytomation; monoclonal, dilution 1: 80). Immunostained sections were interpreted as positive if they showed either a focal or diffuse cytoplasmic reaction product, with nuclear expression in the case of p53 and MIB-1. To quantify the percentage of nuclei staining with MIB-1, a nuclear antigen, at least 500 tumour cells were manually counted three times in the region of tumour with the highest nuclear staining, and an average percentage of positively stained nuclei calculated.

\section{Statistical methods}

Continuous variables and categorical variables with more than three categories were dichotomised on the basis of their median values or the variables' clinical significance-that is, age $(<65$ years, $\geqslant 65$ years), MIB-1 labelling index $(\leqslant 5,>5)$, extent of tumour removal (Simpson grade I, II and $\geqslant$ III) and tumour location (convexity, non-convexity). All the data are expressed with standard errors. Survival was estimated by the KaplanMeier method, and the log-rank test was used for comparisons. A Firth's Cox proportional hazards model was used to analyse possible prognostic factors for the risk of recurrence or death. To assess the effect of adjuvant radiotherapy, the patients were divided into two groups: radiotherapy group and non-radiotherapy group. A two-sided probability level of 0.05 was chosen for statistical significance.

\section{Results}

On application of the 2000 WHO classification system, 10 of 74 meningiomas were reclassified as benign, 40 as atypical and 24 as anaplastic meningioma (table 1). Reassessment of the meningiomas resected before 2001 (meningiomas originally graded by using the 1993 WHO classification system) revealed that $43.8 \%$ (25/57) of high-grade meningiomas were reclassified. Reassessment of the meningiomas resected after January 2001 (meningiomas graded by using the 2000 WHO classification system after January 2001) revealed that $5.8 \%(1 / 17)$ of meningiomas originally classified as grade II were reclassified as grade I.
Brain invasion was evaluated only in 81\% (60/74) of cases where it was assessable. Dissection of the tumour was performed between two membranes of the tumour capsule. Complete resection of tumour could be carried out with ease if a clear arachnoid plane was preserved between the tumour and adjacent brain parenchyma. However, when a clear arachnoid plane disappeared, adjacent brain parenchyma as well as tumour was intentionally removed to assess the brain invasion. The remaining 14 cases without adjacent brain parenchyma were unassessable to brain invasion because the existence of a clear arachnoid plane helped the tumour to be completely resected. These 14 cases did not show tumour recurrence in the last follow-up neuroimaging studies. Of these 60 cases wherein brain parenchyma was identified, 46 (77\%) showed brain invasion. The presence of brain invasion was associated with decreased recurrence-free survival $(p=0.013)$ but had no influence on overall survival ( $p>0.268)$.

The rate of p53 overexpression was observed in 10\% (1/10) of benign meningiomas, $25 \%$ (10/40) of atypical meningiomas, and $79 \%(19 / 24)$ of anaplastic meningiomas, and it became significantly higher as histological malignancy increased $(p<0.001)$. The mean MIB-1 labelling indexes for p53 overexpression-negative and p53 overexpression meningiomas were $3.3 \pm 5.7 \%$ and $8.9 \pm 10.5 \%$, respectively $(p=0.010)$. Overexpression of p53 was detected in $40.5 \%$ (30/74) of meningiomas (15/51 nonrecurrent and 15/23 recurrent). There was a significant correlation between p53 overexpression and tumour recurrence $(p=0.004)$.

As shown in figure 1, overall and recurrence-free survivals were all better for patients with benign compared with atypical meningiomas $(p<0.001)$ and for patients with atypical compared with anaplastic meningioma $(p<0.001)$. The mean MIB-1 labelling indexes for benign, atypical and anaplastic meningiomas were $0.8 \%, 3.2 \%$ and $12.0 \%$, respectively ( $p<0.001$ ) (fig. 2 ).

The 64 patients reclassified as having either atypical or anaplastic meningioma were included in the present analysis. Clinicopathological characteristics of these 64 patients are summarised in table 2 .

The female to male ratio was 2.1:1 for atypical meningioma and $0.5: 1$ for anaplastic meningioma. This lack of a female predominance in anaplastic meningiomas was significant $(p=0.008)$.

\section{Atypical meningioma}

The mean clinical follow-up period was 63.6 months (range, $0.6-154.5)$. The mean overall and mean recurrence-free survivals were $142.5 \pm 6.0$ months and $138.5 \pm 7.0$ months, respectively (fig. 1). Ten-year overall and recurrence-free survival rates were $89.6 \%$ and $87.1 \%$, respectively. In univariate testing, the extent of resection ( $\geqslant$ Simpson grade III) and malignant progression

Table 1 Cross-tabulation of grade change on the basis of histopathological classification system

\begin{tabular}{lllll}
\hline \multirow{2}{*}{ Initial grade } & \multicolumn{4}{l}{ Grade according to the WHO 2000 classification system } \\
\cline { 2 - 5 } & I (Benign) & II (Atypical) & III (Anaplastic) & Total \\
\hline II & $5^{*}$ & 26 & $2^{\dagger}$ & 33 \\
III & $5^{\star}$ & $14^{\S}$ & 22 & 41 \\
Total & 10 & 40 & 24 & 74 \\
\hline
\end{tabular}

\footnotetext{
*These were previously considered to be atypical meningioma only because of a raised MIB-1 labelling index.

These tumours were reclassified based on a mitotic index of more than 20 mitotic figures per 10 high-power fields.

These five patients were previously diagnosed on the basis of the presence of brain invasion rather than obviously malignant cytology.

${ }^{5}$ Thirteen patients were previously diagnosed on the basis of the presence of brain invasion and 1 patient did not fulfill the pathological criteria for anaplastic meningioma.
} 
Figure 1 Kaplan-Meier survival curves were significantly better for patients with benign meningioma compared with atypical meningioma $(p<0.001)$, and for patients with atypical meningioma compared with anaplastic meningioma $(p<0.001)$. (A) Overall survival; (B) Recurrence-free survival.
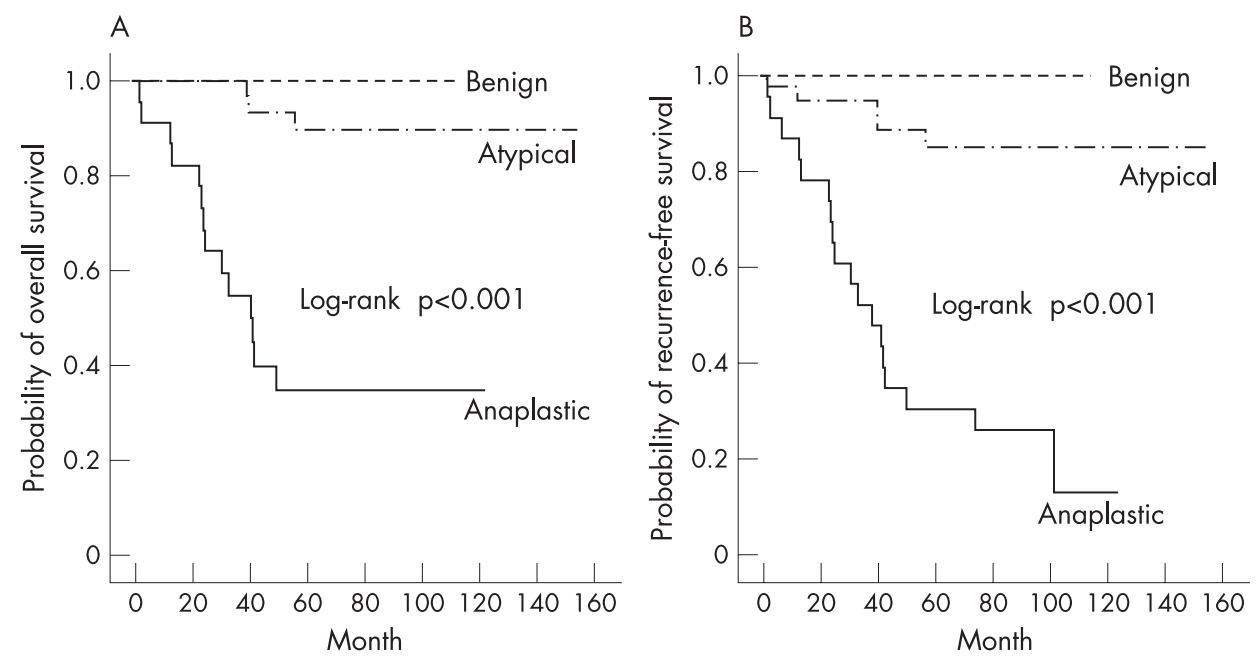

were significant unfavourable prognostic factors for recurrencefree survival. In multivariate analysis, no factor was associated with increased overall survival, except the extent of resection, which had a borderline significance $(p=0.057)$. The extent of resection was significantly associated with reduced recurrencefree survival (OR 2.406; 95\% CI 1.092 to 5.457; $p=0.012$ ). Brain invasion and adjuvant radiotherapy were not associated with patient survival. Moreover, differences between the radiotherapy subgroup and non-radiotherapy subgroup were not significant for overall survival and recurrence-free survival. However, in the radiotherapy subgroup, multivariate analysis identified brain invasion as an independent unfavourable prognostic factor for overall (OR 3.245; 95\% CI 1.896 to $7.065 ; p=0.025)$ and recurrence-free survival (OR 4.125; 95\% CI 2.145 to $9.568 ; p=0.007)$, whereas it had no effect on survival in the non-radiotherapy subgroup. Age, MIB-1 labelling index, tumour location, malignant progression and p53 overexpression had no significant effect on either recurrence-free survival or overall survival.

Of the four patients showing tumour recurrence, three were treated using gamma knife radiosurgery and the other underwent the second operation. At last follow-up, three patients had died as a result of tumour progression.

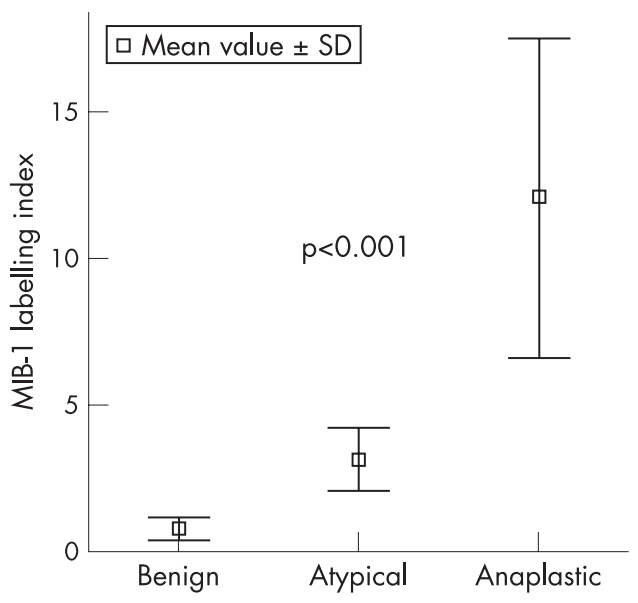

Figure 2 The proliferative marker, MIB-1, is directly correlated with the histological grade of meningiomas $(p<0.001)$. Mean labelling indexes are $0.8 \%$ in benign, $3.2 \%$ in atypical and $12.0 \%$ in anaplastic meningiomas.

\section{Anaplastic meningioma}

The mean clinical follow-up period of the 24 patients with anaplastic meningioma was 42.5 months (range, 0.3-122.6). Of these 24 patients, 17 died and 7 remained alive: 14 patients died from tumour progression, 1 from postoperative bacterial ventriculoencephalitis, 1 from a traffic accident and 1 from acute myocardial infarction. The median overall survival was $39.8 \pm 7.8$ months, and the 3 - and 5-year survival rates were $55 \%$ and $35 \%$, respectively (fig $1 \mathrm{~A}$ ). Tumour recurrence occurred in $75 \%(18 / 24)$ of patients, and $58 \%(14 / 24)$ of patients died from tumour progression at a median of 5.1 months, with spinal metastases developing in 3 of the 18 patients. The median recurrence-free survival was $32.2 \pm 8.0$ months. The 3 - and 5year recurrence-free survival rates were $50 \%$ and $29 \%$, respectively (fig 1B). Multivariate analysis identified brain invasion, adjuvant radiotherapy, malignant progression, p53 overexpression and extent of resection as independent prognostic factors for survival (table 3).

Of the 18 patients showing tumour recurrence, 5 patients with 6 recurrent anaplastic meningiomas were treated using gamma knife radiosurgery. The median tumour volume was $6.3 \mathrm{ml}$ (range, 0.6-36.8) and the median margin dose was $13 \mathrm{~Gy}$ (range, 10-21). The median neuroradiological follow-up period was 14 months (range, 8-34). Four of 6 tumours showed a local failure and $80 \%(4 / 5)$ of patients died from tumour recurrence at the last follow-ups. One patient with tumour recurrence received five courses of chemotherapy with carmustine, but he died of tumour progression. Of the three patients with spinal metastases, two underwent surgical resection followed by radiotherapy and the other received radiotherapy only. These 3 patients did not show tumour recurrence in the last follow-up spinal neuroimaging studies.

\section{Malignant progression}

Among the 64 patients with atypical or anaplastic meningiomas, 20 showed a histopathological progression towards a higher grade that was associated with an aggressive clinical course. Among these 20 meningiomas, 7 atypical and 10 anaplastic meningiomas were transformed from benign meningiomas, and 3 anaplastic meningiomas from atypical meningiomas. The risk of death was significantly higher for patients with malignant progression than for patients without malignant progression (OR 13.507; 95\% CI 2.015 to 90.556; $p=0.007$ ) (fig $3 \mathrm{~A}$ ). The risk of recurrence was also higher for the patients 
Table 2 Clinicopathological characteristics of patients with atypical or anaplastic meningioma

\begin{tabular}{|c|c|c|}
\hline \multirow[b]{2}{*}{ Characteristics } & \multicolumn{2}{|l|}{ Value } \\
\hline & Atypical & Anaplastic \\
\hline Patients (number) & 40 & 24 \\
\hline Age at diagnosis (years) & $49.0 \pm 13.3$ (range, $17-75)$ & $51.1 \pm 16.0$ (range, $17-77)$ \\
\hline Male/female ratio & $13 / 27$ & $16 / 8$ \\
\hline Karnofsky performance score & 80 (range, 40-100) & 80 (range, 60-100) \\
\hline Parasagittal & 10 & 6 \\
\hline Sphenoid ridge & 5 & 3 \\
\hline Falx & 3 & 4 \\
\hline Tuberculum sellae & 2 & 1 \\
\hline Intraventricular & 2 & 1 \\
\hline Tentorial & 2 & 1 \\
\hline \multicolumn{3}{|c|}{ Extent of resection (Simpson grading system ${ }^{5}$ ) } \\
\hline Grade I & 16 & 6 \\
\hline Grade II & 14 & 2 \\
\hline Grade III & 4 & 8 \\
\hline Grade IV & 6 & 4 \\
\hline Grade V & 0 & 4 \\
\hline Adjuvant radiotherapy & 23 & 17 \\
\hline Adjuvant chemotherapy & 0 & 1 \\
\hline Palliative gamma knife radiosurgery & 3 & 5 \\
\hline Extracranial metastasis & 0 & 3 (spine) \\
\hline MIB-1 labelling index (\%) & $3.2 \pm 3.0$ (range, $0.5-10.0$ ) & $12.0 \pm 11.6$ (range, $0.5-38.0$ ) \\
\hline Brain invasion & $20(50 \%)$ & $21(88 \%)$ \\
\hline
\end{tabular}

Values are expressed as mean \pm SD.

with malignant progression (OR 4.793; 95\% CI 1.614 to 14.239 ; $\mathrm{p}=0.005$ ) (fig $3 \mathrm{~B}$ ). The mean period for malignant progression was 70.0 months (range 10.7 to 118.3) from benign to atypical meningiomas, 89.7 months (range 10.8 to 152.2 ) from benign to anaplastic meningiomas, and 39.8 months (range 13.5 to 62.5 ) from atypical to anaplastic meningiomas. Multivariate analysis of these 20 tumours showed that p53 overexpression was the only independent prognostic factor for malignant progression (OR 5.753; 95\% CI 1.551 to 21.329; $p=0.009$ ).

The patients with malignant progression (75\%) (52\%, 80\% and $100 \%$ in benign to atypical, benign to anaplastic, and atypical to anaplastic, respectively) had a significantly higher percentage of p53 overexpression than the patients without malignant progression $(32 \%)(p=0.001)$. The mean MIB-1 labelling indexes in the progression group and the nonprogression group were $8.0 \pm 10.1 \%$ and $5.9 \pm 8.1 \%$, respectively, but lacked statistical power. Tumours with malignant progression resulted in $35 \%(7 / 20)$ of atypical meningiomas and $65 \%$ $(13 / 20)$ of anaplastic meningiomas. On the other hand, tumours without malignant progression resulted in $75 \%(33 / 44)$ of atypical and $25 \%(11 / 44)$ of anaplastic patients. The proportion of anaplastic meningioma among the tumours with malignant progression was significantly higher than that among tumours without malignant progression $(p<0.001)$. Tumour recurrence was observed in $70 \%(14 / 20)$ of the patients with malignant progression and $18 \%(8 / 44)$ of the patients without malignant progression. There was a significant correlation between malignant progression and tumour recurrence $(p<0.001)$.

Table 3 Potential prognostic factors for survival in anaplastic meningioma

\begin{tabular}{|c|c|c|c|c|}
\hline \multirow[b]{2}{*}{ Survival factors } & \multicolumn{2}{|c|}{ Overall survival } & \multicolumn{2}{|c|}{ Recurrence-free survival } \\
\hline & p Value & Odds ratio $(95 \% \mathrm{Cl})$ & p Value & Odds ratio $(95 \% \mathrm{CI})$ \\
\hline Brain invasion & 0.002 & $8.226(2.251-30.026)$ & $<0.001$ & $9.157(2.699-31.061)$ \\
\hline Adjuvant radiotherapy & 0.002 & $0.006(0.000-0.150)$ & $<0.001$ & $0.004(0.000-0.085)$ \\
\hline Malignant progression & 0.021 & $10.262(2.753-52.144)$ & 0.019 & $8.140(1.981-33.481)$ \\
\hline Extent of resection (I, II/III, IV, V) & 0.001 & $2.529(1.205-5.309)$ & 0.018 & $2.121(1.140-3.949)$ \\
\hline p53 overexpression & 0.034 & $3.019(1.725-8.652)$ & 0.026 & $2.878(1.549-7.818)$ \\
\hline Sex (male/female) & 0.926 & $0.848(0.027-27.005)$ & 0.098 & $0.904(0.587-2.233)$ \\
\hline Age $(\geqslant 65 /<65$ years $)$ & 0.654 & $0.967(0.836-1.119)$ & 0.300 & $0.967(0.907-1.031)$ \\
\hline Tumour location (non-convexity/convexity) & 0.090 & $0.514(0.332-5.907)$ & 0.210 & $0.733(0.155-3.460)$ \\
\hline MIB-1 labelling index $(\leqslant 5 />5)$ & 0.541 & $1.033(0.201-8.269)$ & 0.062 & $1.486(0.981-2.250)$ \\
\hline
\end{tabular}


Figure 3 Overall survival $(A)$ and recurrence-free survival $(B)$ were significantly different between patients with malignant progression and those without malignant progression $(p=0.007$ and $p=0.005$, respectively).
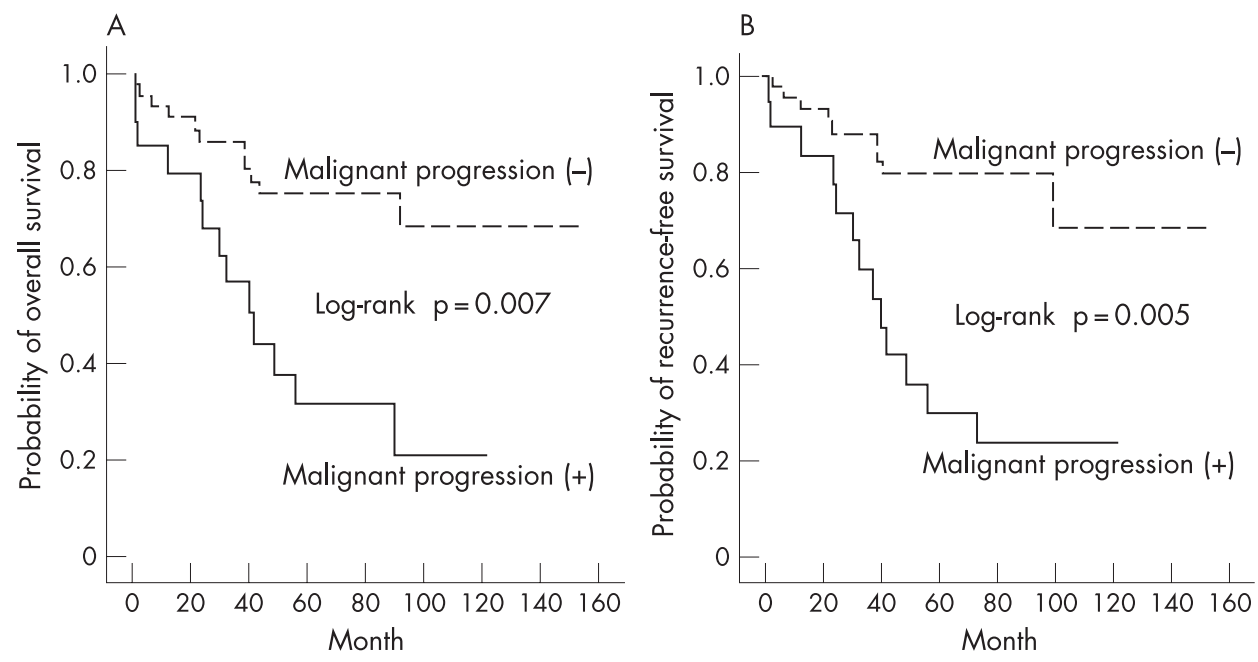

\section{DISCUSSION}

\section{Proliferation index and brain invasion}

Although a poor prognosis may be associated with a high MIB-1 labelling index, significant overlap exists in the MIB-1 labelling ranges for benign, atypical and anaplastic meningiomas. ${ }^{6}$ Moreover, interinstitutional and interobserver variation has been reported in meningioma grading. Therefore, MIB-1 labelling cannot be a single parameter to establish meningioma grade in the $2000 \mathrm{WHO}$ classification. However, our study also showed a correlation between MIB-1 labelling index and the histological grade of the meningiomas. These results are consistent with those of previous reports. ${ }^{89}$

Brain invasion has long been considered a worrisome feature in meningioma resection specimens, but it has been debated whether brain invasion constitutes a single criterion of malignancy. Recent molecular genetic investigations have failed to show genetic changes that are characteristic of non-benign meningiomas in histologically benign meningiomas that display brain invasion. ${ }^{10}{ }^{11}$ Furthermore, the presence of brain invasion does not correlate with an aggressive course of anaplastic meningioma and only increases the likelihood of recurrence such as that of atypical meningioma, not anaplastic meningioma. Like some other studies, ${ }^{12-14}$ our study revealed that the presence of brain invasion was a powerful predictor of reduced recurrence-free survival, but the worst prognosis had a close relationship with meningiomas with frank histological anaplasia, whether invasive or not.

\section{Atypical meningioma}

Surgery has been the primary treatment modality for meningiomas, regardless of subtype or grade. Similar to benign meningiomas, gross total resection of an atypical meningioma is associated with lower recurrence rates and increased survival than with subtotal resection. ${ }^{15}$ Our study showed similar results: Simpson Grade I, II resection without adjuvant radiotherapy might be sufficient to achieve durable local control and a longer survival period. Furthermore, adjuvant radiotherapy did not improve patient survival, regardless of the extent of resection. However, this result should be interpreted with caution. Invasive meningiomas are often adherent or intertwined with cortical vessels and therefore more difficult to excise. ${ }^{16}$ Moreover, microscopic brain invasion emerged as the most powerful predictor of reduced recurrence-free survival. ${ }^{12}$ Adjuvant radiotherapy contributed significantly to improvement in overall survival and recurrence-free survival in the brain-invasive meningioma subgroup in our study. Several studies have demonstrated that adjuvant radiotherapy improves overall and recurrence-free survival in atypical meningiomas following incomplete tumour resection. ${ }^{14-18}$ Based on the above description, if the atypical meningioma is completely resected (Simpson Grade I, II) and does not reveal brain invasion, we do not recommend adjuvant radiotherapy. However, if the atypical meningioma was incompletely resected or showed brain invasion, adjuvant radiotherapy may be helpful for improved patient outcome.

\section{Anaplastic meningioma}

Until recently, there have been no reports about treatment paradigm using the 2000 WHO classification on anaplastic meningioma. Nevertheless, two suggestions about treatment strategies exist: (1) radiotherapy is indispensable in anaplastic meningioma regardless of the extent of resection, ${ }^{15}{ }^{16}{ }^{19}$ and (2) a complete resection for an anaplastic meningioma leads to reduced recurrence and increased survival. ${ }^{12} 20$ The treatment outcome of patients reported in our study is consistent with these findings.

In our study, extent of resection, adjuvant radiotherapy, brain invasion and malignant progression were significantly associated with survival and recurrence. Brain invasion, in particular, was an adverse prognostic factor for tumour recurrence, as reported in the previous studies, ${ }^{13}$ and associated with a significant decrease in survival in our study. Furthermore, most of the patients (88\%) with tumour recurrence died from tumour progression. Malignant progression has not been previously identified as a survival factor in a meningioma study. However, it was a significantly unfavourable prognostic factor for survival in our study.

\section{p53 overexpression and malignant progression}

Some studies showed that overexpression of $\mathrm{p} 53$ by genetic mutation or protein stabilisation might result in unrestricted tumour growth ${ }^{21}$ and overexpression of p53 in meningiomas was associated with increased proliferation marker MIB-1, recurrence and a positive correlation with the histological grades of meningiomas. ${ }^{69} 922-24$ Our study also showed similar results to the above studies. This factor may be valuable in determining the grade of meningiomas in histologically controversial cases. 
Our study showed that $1.8 \%$ of the 1098 patients experienced malignant progression from previous histology. This result is similar to the previously reported rates of $0.16-2 \%$ of all meningiomas and the rate of malignant progression in recurring tumours is higher in atypical than benign meningiomas, ${ }^{4} 182025$ which supports our results. The elapsed period to the malignant progression ranged from 8 months to 26 years. ${ }^{42627}$ In our study, it took about 70.0 months to progress to atypical meningioma and 89.7 months to progress to anaplastic meningioma from benign meningioma, and it was shorter (39.8 months) to progress to an anaplastic tumour from an atypical one.

It is still a matter of controversy whether the prognosis of patients with secondary glioblastoma is similar to or better than that of patients with primary lesions. ${ }^{28}$ In contrast to the glioblastomas, the prognosis of patients with malignant progression was worse than that of patients with primary high-grade meningiomas in our study. Compared with glial tumours, little is known about the molecular pathogenesis of meningiomas and their malignant progression. Several studies showed that a number of cytogenetic alterations were associated with malignant progression, and these cytogenetic alterations might also be markers of malignant potential that might influence tumour recurrence and poor prognosis. ${ }^{4011242930}$ Amatya et al reported that p53, as an apoptosis-related protein, is likely to be important in not tumorigenesis but malignant progression or recurrence. Overexpression of p53, which is probably a surrogate for p53 mutations, ${ }^{23} 26$ was also the major predicting factor for malignant progression in our study. These results are consistent with those of previous reports..$^{81}$ Therefore, we recommend that meningiomas with p53 overexpression should be observed extremely closely with radiographic follow-up.

\section{Treatment for recurrent high-grade meningioma}

Relatively small numbers of published studies have defined the role of stereotactic radiosurgery for atypical and anaplastic meningiomas. Harris et al ${ }^{32}$ recently reported that the 5-year survival rate was $59 \%$ and the local control rate at 5 years was $83 \%$ for atypical meningiomas in 18 patients who had previously undergone surgical resection. Stafford et al..$^{33}$ also showed that 5-year survival and local control rates for atypical meningioma in 13 patients were 76 and $68 \%$, respectively. These studies showed that stereotactic radiosurgery may have a place as an adjunct to treatment for residual or recurrent atypical meningiomas. However, the effectiveness of stereotactic radiosurgery for anaplastic meningioma was controversial. Five-year survival and local control rates for anaplastic tumours were reportedly $59 \%$ and $72 \%$ in one study, ${ }^{32}$ and $0 \%$ and $0 \%$ in another study, ${ }^{33}$ respectively. In our study, although $80 \%$ of patients died of tumour recurrence within 20 months after gamma knife radiosurgery, one showed no evidence of tumour recurrence for 34 months after gamma knife radiosurgery. Thus, stereotactic radiosurgery may play a role in therapy, but further study and larger numbers of patients are required.

Recently, the role of hydroxyurea in the treatment of recurrent high-grade meningiomas has been explored. ${ }^{34-36}$ The authors reported that one anaplastic meningioma responded to hydroxyurea, whereas 8 (seven atypical, one anaplastic) did not. In our study, carmustine could not arrest the growth of anaplastic meningioma. Two forms of therapies are for salvage purposes and should only be used when the patient is no longer a candidate for further current standard therapy.

\section{Limitations of the current study}

The data for our study were collected retrospectively and thus a potential for bias may exist. Moreover, the small cohort of study group may not be well suited to an assessment of our treatment policy. The gold standard for evaluating our treatment policy is a prospective randomised trial, but this approach is not possible due to the rarity of high-grade meningiomas.

\section{Conclusions}

We have found that a precise meningioma grading system may help to avoid the over-treatment of patients with an atypical meningioma and that, once the tumour has "declared itself" by recurrence and upgrading histological features, it becomes a tumour that is poorly amenable to current therapies. As it is accepted that atypical and anaplastic meningiomas evolve by the accumulation of cytogenetic aberrations and that subsequent genomic changes result in a poorer clinical outcome, the 2000 WHO classification should be improved using a renewed framework that considers genetic studies as part of meningioma diagnosis.

\section{Competing interests: None.}

\section{REFERENCES}

1. Cushing HW, Eisenhardt L. Meningiomas: Their Classification, Regional Behavior, Life History, and Surgical End Results. Springfield, Illinois: Charles C Thomas, 1938.

2. Louis D, Scheithauer B, Budka H, et al. Meningiomas. In: Kleihues P, Cavenee W, eds. World Health Organization Classification of Tumours: Pathology and Genetics of Tumours of the Nervous System. Lyon: IARC Press, 2000:176-84.

3. Nowell PC. Tumor progression: a brief historical perspective. Semin Cancer Biol 2002;12:261-6.

4. Al-Mefty 0, Kadri PA, Pravdenkova S, et al. Malignant progression in meningioma: documentation of a series and analysis of cytogenetic findings. J Neurosurg 2004;101:210-8.

5. Simpson D. The recurrence of intracranial meningiomas after surgical treatment. J Neurol Neurosurg Psychiatry 1957;20:22-39.

6. Karamitopoulou E, Perentes E, Tolnay M, et al. Prognostic significance of MIB-1, p53, and bcl-2 immunoreactivity in meningiomas. Hum Pathol 1998;29:140-5.

7. Kleihues $\mathbf{P}$, Louis DN, Scheithauer BW, et al. The WHO classification of tumors of the nervous system. J Neuropathol Exp Neurol 2002;61:215-25; discussion 26-9.

8. Amatya VJ, Takeshima Y, Sugiyama K, et al. Immunohistochemical study of Ki-67 (MIB-1), p53 protein, p21WAF1, and p27KIP1 expression in benign, atypical, and anaplastic meningiomas. Hum Pathol 2001;32:970-5.

9. Perry A, Stafford SL, Scheithauer BW, et al. The prognostic significance of MIB-1, p53, and DNA flow cytometry in completely resected primary meningiomas. Cancer 1998;82:2262-9.

10. Weber RG, Bostrom J, Wolter M, et al. Analysis of genomic alterations in benign, atypical, and anaplastic meningiomas: toward a genetic model of meningioma progression. Proc Natl Acad Sci USA 1997;94:14719-24.

11. Simon M, von Deimling A, Larson JJ, et al. Allelic losses on chromosomes 14, 10, and 1 in atypical and malignant meningiomas: a genetic model of meningioma progression. Cancer Res 1995;55:4696-701.

12. Perry A, Scheithauer BW, Stafford SL, et al. "Malignancy" in meningiomas: a clinicopathologic study of 116 patients, with grading implications. Cancer 1999;85:2046-56.

13. Perry A, Stafford SL, Scheithauer BW, et al. Meningioma grading: an analysis of histologic parameters. Am J Surg Pathol 1997;21:1455-65.

14. Coke CC, Corn BW, Werner-Wasik M, et al. Atypical and malignant meningiomas: an outcome report of seventeen cases. J Neurooncol 1998;39:65-70.

15. Dziuk TW, Woo S, Butler EB, et al. Malignant meningioma: an indication for initial aggressive surgery and adjuvant radiotherapy. J Neurooncol 1998;37:177-88.

16. Modha A, Gutin PH. Diagnosis and treatment of atypical and anaplastic meningiomas: a review. Neurosurgery 2005;57:538-50; discussion: 538-50.

17. Milosevic MF, Frost PJ, Laperriere NJ, et al. Radiotherapy for atypical or malignant intracranial meningioma. Int J Radiat Oncol Biol Phys 1996;34:817-22.

18. Jaaskelainen J, Haltia M, Servo A. Atypical and anaplastic meningiomas: radiology, surgery, radiotherapy, and outcome. Surg Neurol 1986;25:233-42.

19. Hug EB, Devries A, Thornton AF, et al. Management of atypical and malignant meningiomas: role of high-dose, 3D-conformal radiation therapy. $J$ Neurooncol 2000;48:151-60.

20. Palma L, Celli P, Franco $\mathrm{C}$, et al. Long-term prognosis for atypical and malignant meningiomas: a study of 71 surgical cases. J Neurosurg 1997;86:793-800.

21. Louis DN. The p53 gene and protein in human brain tumors. J Neuropathol Exp Neurol 1994;53:11-21. 
22. Lanzafame S, Torrisi A, Barbagallo G, et al. Correlation between histological grade, MIB-1, p53, and recurrence in 69 completely resected primary intracranial meningiomas with a 6 year mean follow-up. Pathol Res Pract 2000;196:483-8.

23. Nagashima G, Aoyagi M, Yamamoto M, et al. p53 overexpression and proliferative potential in malignant meningiomas. Acta Neurochir (Wien) 1999;141:53-61; discussion $0-1$.

24. Ohkoudo M, Sawa H, Hara M, et al. Expression of p53, MDM2 protein and Ki-67 antigen in recurrent meningiomas. J Neurooncol 1998;38:41-9.

25. Jaaskelainen J, Haltia M, Laasonen $\mathrm{E}$, et al. The growth rate of intracranial meningiomas and its relation to histology. An analysis of 43 patients. Surg Neurol 1985;24:165-72.

26. Matsuno A, Fujimaki T, Sasaki T, et al. Clinical and histopathological analysis of proliferative potentials of recurrent and non-recurrent meningiomas. Acta Neuropathol (Berl) 1996;91:504-10.

27. Yamazaki Y, Kawano N, Suwa T, et al. [Recurrent meningioma with malignant transformation: a case which changed from meningothelial type to papillary type]. No Shinkei Geka 1994;22:285-9.

28. Dropcho EJ, Soong SJ. The prognostic impact of prior low grade histology in patients with anaplastic gliomas: a case-control study. Neurology 1996;47:684-90.
29. Lusis E, Gutmann DH. Meningioma: an update. Curr Opin Neurol 2004;17:687 $-92$.

30. Perry A, Gutmann DH, Reifenberger G. Molecular pathogenesis of meningiomas. J Neurooncol 2004;70:183-202.

31. Cho H, Ha SY, Park SH, et al. Role of p53 gene mutation in tumor aggressiveness of intracranial meningiomas. J Korean Med Sci 1999;14:199-205.

32. Harris AE, Lee JYK, Omalu B, et al. The effect of radiosurgery during management of aggressive meningiomas. Surg Neurol 2003;60:298-305.

33. Stafford SL, Pollock BE, Foote RL, et al. Meningioma radiosurgery: Tumor control, outcomes, and complications among 190 consecutive patients. Neurosurgery 2001:49:1029-37.

34. Loven $\mathbf{D}$, Hardoff $\mathrm{R}$, Sever ZB, et al. Non-resectable slow growing meningiomas treated by hydroxyurea. J Neurooncol 2004;67:221-6.

35. Mason WP, Gentili F, MacDonald DR, et al. Stabilization of disease progression by hydroxyurea in patients with recurrent or unresectable meningioma. J Neurosurg 2002;97:341-6.

36. Schrell UMH, Rittig MG, Anders M, et al. Hydroxyurea for treatment of unresectable and recurrent meningiomas: ॥-Decrease in the size of meningiomas in patients treated with hydroxyurea. J Neurosurg 1997;86:840-4.

\section{Save your favourite articles and useful searches}

Use the "My folders" feature to save and organise articles you want to return to quickly-saving space on your hard drive. You can also save searches, which will save you time. You will only need to register once for this service, which can be used for this journal or all BMJ Journals, including the BMJ. 